\title{
Del estructuralismo al cognitivismo: hacia un enfoque cientifista de la narratología.
}

Jesús JIMÉNEZ VAREA

Antonio PINEDA CACHERO

(Universidad de Sevilla)

http://dx.doi.org/10.12795/AdMIRA.2009.01.10

\begin{abstract}
Resumen: Este artículo intenta realizar una reflexión metateórica sobre algunas relaciones que pueden establecerse entre la narratología estructuralista y la narratología cognitiva. Se ofrecen panorámicas sintéticas sobre cada uno de estos enfoques narratológicos, y se enfatiza particularmente el componente cientifista del estructuralismo.
\end{abstract}

Palabras clave: Narratología estructural, narratología cognitiva, estructuralismo, ciencias cognitivas.

\begin{abstract}
This paper aims to advance a metatheoretical reflection on some relations that can be established between structuralist narratology and cognitive narratology. It provides succinct and panoramic reviews of both narratological approaches, particularly highlighting the scientifist component of structuralism.
\end{abstract}

Keywords: Structural Narratology, Cognitive Narratology, Structuralism, Cognitive Sciences.

\section{Introducción}

El objetivo de este artículo es realizar una reflexión de naturaleza metateórica acerca de los puntos de contacto que pueden establecerse entre la narratología estructuralista y la narratología cognitiva, centrándonos particularmente en la hipótesis de que existe una correspondencia isomórfica entre las estructuras narrativas textuales (objetivo clásico del estructuralismo) y las estructuras y sistemas mentales de procesamiento informativo en formato narrativo. Apuntamos también el potencial que posee la idea de un enfoque neurofisiológico de la narratología, que entendemos como un paso necesario si se adopta un enfoque cognitivista en el estudio de los mensajes narrativos. 


\section{La narratología estructural}

Cuando Tzvetan Todorov acuñó el témino narratologie en su Grammaire $d u$ Décameron para denominar a la "science du récit" (1969: 10), lo hizo para referirse a las iniciativas que, en ese sentido, venían desarrollando algunos de los semiólogos más destacados del ámbito francófono. Podría situarse el inicio "oficial” de esta corriente en la publicación del octavo número de Communications (1966), dedicado precisamente al análisis del relato y al que contribuyeron muchas de aquellas destacadas figuras, que marcarían el desarrollo de las teorías narrativas durante décadas: el mismo Todorov, Roland Barthes, Claude Bremond, Christian Metz, Gerard Genette, Algirdas Julien Greimas y Umberto Eco. La colección de artículos resultantes constituye una especie de manifiesto programático para un estudio estructural e intrínseco del discurso narrativo en el que se reconoce la deuda - a la vez que se establece el imperativo de situarse en continuidad- con la tradición estructuralista derivada del fundamental Cours de linguistique générale (1916) de Ferdinand de Saussure.

En concreto, en el artículo que abre el mencionado volumen de Communications, "Introduction à l'analyse structurale des récits", Barthes reclama para la lingüística saussureana el estatus de ciencia-piloto en función de la cual dotar de una lógica estructural a un análisis decronologizado -esto es, sincrónico- del relato que tuviese como último nivel posible de aplicación el de la narración, más allá del cual los sistemas de elementos extratextuales de carácter histórico, social, ideológico, etc., hacen necesario recurrir a otras semióticas (cfr. 1969: 1-27). Así, la aún sin bautizar Narratología se inscribía en el marco más amplio del Estructuralismo, que tan importantes repercusiones tenía en el campo general de las ciencias sociales y las humanidades. La narratología, por consiguiente, se desarrolló en el contexto del movimiento estructuralista (cfr. Ryan, 1979: 127; Meister, 2003: 201).

Millner y Browitt describen esta macrocorriente de pensamiento de una manera lo suficientemente sintética y general como "an approach to the study of human culture centred on the search for constraining patterns, or structures, which claimed that individual phenomena have meaning by virtue of their relation to other phenomena as elements within a systematical structure" (2002: 93). Aunque el término estructura 
como tal apenas aparecía en su tratado de lingüística, De Saussure introdujo esta distinción fundamental entre los fenómenos individuales y la estructura sistemática subyacente a todos ellos, que está en la base de todo planteamiento estructuralista, al diferenciar dentro de la language entre cada uno de sus usos particulares y el código general al que todos ellos responden necesariamente: la parole vs. la langue. La generalización de los términos "estructura", y más tarde "estructuralismo", se debería al círculo de lingüistas checos conocidos como la Escuela de Praga, con Nikolái Trubetzkoy y Roman Jakobson a la cabeza, cuyo manifiesto de 1930 establece como propósito general del grupo "to work on the basis of functional-structural method toward progress in linguistic research" (en Toman, 1995: 265).

A su vez, Jakobson procedía de la escuela de teoría literaria de los formalistas rusos, quienes habían hecho de la narrativa en un sentido amplio su objeto específico de estudio e, influidos por los trabajos sobre composición narrativa de la poética morfológica alemana ${ }^{1}$, se habían dedicado a teorizar niveles superiores de la estructura narrativa mediante un proceso de abstracción e idealización a partir de numerosos casos particulares. Entre los formalistas, una influencia especialmente poderosa sobre los narratólogos del entorno estructuralista fue Vladimir Propp, con su Morfología del cuento maravilloso ([1928] 1998), al distinguir, a la manera de la lingüística saussureana, entre las componentes variables del relato que se manifiestan en cuentos particulares y las invariantes que corresponden a las estructuras narrativas de orden superior. Su diferenciación de los mutables dramatis personae frente al número finito de acciones que pueden desempeñar en la trama y las resultantes esferas de acción supone un precedente directo del análisis proto-narratológico del Mito de Edipo que ejecutaría el antropólogo estructuralista Claude Lévi-Strauss (1958), así como del modelo de actantes desarrollado por Greimas (1966). Asimismo, Boris Tomashevsky ([1925] 1965) perfila la distinción entre bound vs. free motifs (motivos relevantes frente a otros irrelevantes para la trama), que inspira modelos similares en las teorías narrativas de Barthes (núcleos vs. catalizadores, en el ya citado artículo de 1966) y del narratólogo nortemericano Seymour Chatman (kernells vs. satellites, 1978: 53-6). Y de estas consideraciones sobre lo que integra el esqueleto puro de la trama surge la oposición fundamental fabula vs. siuzhet, introducida en primer lugar por Viktor

\footnotetext{
${ }^{1}$ Figuras como Otmar Schissel von Fleschenberg, el germanista Berard Seuffert y Wilhelm Dibelius adelantaron muchos de los puntos tratados después por los formalistas rusos e, incluso mucho más tarde, por los narratólogos franceses (cfr. Dolezel, 1990: 126-141).
} 
Shklovsky ([1929] 1990) y que, renombrada histoire vs. discours por Todorov (1966a) en su contribución al volumen fundacional de la narratología, constituye un elemento clave de esta corriente de teoría narrativa.

Las oposiciones enumeradas más arriba son ejemplos claros de las consecuencias comunes a todo el estructuralismo de haber adoptado el paradigma lingüístico de Saussure, de acuerdo con el cual la oposición binaria está en la base de todo lenguaje, pues la significación surge de la relación de este tipo que se establece entre signos diferentes. Sin embargo, no es determinante en dicho proceso de significación a qué objetos se refieran los signos, por lo que la postura estructuralista ignora la función referencial de los signos en el lenguaje. En este sentido, la narratología, por su carácter estructural, se distancia de la crítica, dado que no se propone interpretar, esto es, determinar lo que significan los sistemas de signos organizados narrativamente, sino cómo significan en cuanto narrativos. Es una parte necesaria de haber tomado como modelo la teoría lingüística, tal como reflexiona Jonathan Culler al cerrar el primer capítulo de su Structuralist Poetics, "The Linguistic Foundation": "Linguistics is not hermeneutic. It does not discover what a sequence means or produce a new interpretation of it but tries to determine the nature of the system underlying the event" (1975: 31).

En esta búsqueda de la langue narrativa, los narratólogos también hicieron suyos los métodos de investigación de la lingüística a la hora de construir sus modelos. Si los lingüistas habían establecido los niveles de descripción fonológico, morfológico y sintáctico, Todorov propuso la ya mencionada segregación entre "historia" y "discurso", y Barthes, desde su visión del discurso narrativo como el objeto de una segunda lingüística, distinguió los niveles de las funciones (en el que se encuentran las aportaciones seminales de Propp y la lógica de los posibles narrativos de Bremond, 1966), de las acciones (aproximadamente el de los actantes de Greimas) y de la narración (cercano al concepto de "discurso" de Todorov) (cfr. Barthes, 1966). De la misma manera, los modelos narratológicos toman prestadas las tradicionales categorías gramaticales y, así, por ejemplo, Todorov lo hace explícito en el título de una obra donde asocia a los agentes y las entidades narrados con los nombres, a los acontecimientos con los verbos y a las propiedades con los adjetivos (cfr. 1969: capítulo II.3). Para acabar con estos ejemplos de apropiación de los fines y de los métodos lingüísticos, Genette establece tres planos de comunicación narrativa - historia, relato y 
narración- a los que aplica un modelo de análisis construido sobre las categorías de tiempo, modo y voz (cfr. "Discours du récit" en Genette, 1972).

Retomando el principio del presente epígrafe, Todorov acuñó el término narratologie para referirse a la teoría narrativa de índole estructuralista, caracterizada por algunos de los rasgos expuestos en los párrafos anteriores. Sin embargo, el término se ha extendido en la actualidad a una multitud de aproximaciones al estudio de la narrativa, muchas de las cuales colisionan frontalmente con los presupuestos epistemológicos del estructuralismo. Dado su énfasis en la estructura, los pensadores estructuralistas en general mostraron desinterés por la agencia humana, los intereses individuales o compartidos y el concepto de comunidad, y, en consecuencia, tuvieron una actitud crítica hacia el empirismo y el humanismo, así como hacia las corrientes de pensamiento que se movían en esos planos, tales como la fenomenología y el existencialismo. Pero, del mismo modo que autores posteriores a Saussure -J. L. Austin, H. P. Grice, J. Searle- se unieron en alguna medida a la proclamación "the meaning of a word is its use in the language" del Wittgenstein tardío (en Luntley, 2003: 50), el contexto y los usos particulares volvieron a introducirse en las teorías narrativas. Así han surgido modalidades ajenas al estructuralismo que constituyen en rigor teorías crítico-interpretativas con algún tipo de orientación contextualista y/o ideológica relacionada con tendencias como el psicoanálisis, el feminismo, el post-colonialismo o los estudios culturales. Al mismo tiempo, entre otros autores, Kimdt y Muller (2003: vi) o Fludernik (2009: 12) han localizado un punto de inflexión en la evolución de las teorías sobre la narrativa como consecuencia de un fenómeno que han llamado "the narrativist turn" (2003: vi), consistente en la importación de los modelos narrativos por parte de disciplinas como la teología, la psicología, la sociología, la historia o el derecho. Pese a que frecuentemente son incompatibles con el espíritu estructuralista que originalmente animara a la narratología, especialistas tan relevantes en la actualidad como David Herman (cfr. 2009: 30-31) han optado por un uso generoso de este término, refiriéndose a la narratología estructural como clásica y englobando entre las postclásicas a las aproximaciones que han proliferado en las décadas posteriores: formalistas, dialógicas, fenomenológicas, aristotélicas, tropológicas, reconstructivas, 
cognitivistas, constructivistas, historicistas, sociológicas, antropológicas, feministas, queer, políticas... ${ }^{2}$

\section{La narratología cognitiva}

Entre las llamadas narratologías post-clásicas, en cierto modo la Narratología Cognitiva se establece en continuidad con la clásica por cuanto que recupera nociones relativas a la cognición humana que estaban en alguna medida presentes en los fundamentos del estructuralismo y de la morfología. En este sentido, Monika Fludernik, cuyos trabajos incurren en esta línea de investigación narrativa, ha hablado de "The Cognitivist Turn and the Resurrection of the Linguistic Model" (2005: 48), como última fase hasta la fecha de una evolución de la narratología durante el siglo XX y hasta la actualidad que bien podría formularse en función de la adopción de sucesivos paradigmas con origen en la lingüística: el estructuralista de la narratología clásica; el generativista de las gramáticas textuales; el semántico-pragmático de la teoría de los actos del habla; el textual del análisis conversacional y el análisis crítico del discurso; y finalmente el paradigma cognitivista de la narratología cognitiva (cfr. ibidem) ${ }^{3}$. Si la lingüística cognitiva pretende dilucidar de qué maneras la cognición humana predetermina la estructura de la lengua, su contrapartida narratológica busca hacer lo propio en relación con las estructuras de los discursos narrativos. En este sentido, en la narratología cognitiva confluyen de algún modo tres campos de estudio: la teoría narrativa, la lingüística y el grupo inter- y multidisciplinar que se ha dado en llamar Ciencias Cognitivas ${ }^{4}$.

En consonancia con la correlación entre lingüística y narrativa, el giro de la narratología hacia el cognitivismo puede observarse ya en la fase estructuralista como consecuencia de los intentos de desarrollar gramáticas narrativas por parte de Barthes (1966), Todorov (1969) y -aunque no la llama grammaire sino logique- Bremond

\footnotetext{
${ }^{2}$ Para obtener una panorámica detallada de estas narratologías post-clásicas, véanse el volumen editado por Phelan y Rabinowitz (2005) y las obras de Fludernik (2005, 2009).

${ }^{3}$ Para la evolución de la narratología desde el formalismo estructuralista hasta la semántica generativa, pasando por la gramática generativa, cfr. Ryan, 1979.

${ }^{4}$ La que podría considerarse una de sus biblias, The MIT Enciclopedia of the Cognitive Sciences, establece seis campos de conocimiento en la fundación de las ciencias cognitivas: la filosofía; la psicología: las neurociencias; la inteligencia computacional; la lingüística y el lenguaje; la cultura, la cognición y la evolución (cfr. Wilson y Keil, 1999: xiii).
} 
(1966, 1973). Influido directamente por estas iniciativas, Gerald Prince reconoce, no obstante, que están abocadas al fracaso por la incapacidad de sus predecesores a la hora de identificar "with precision the basic structural units of a story" (1973: 11). Por ello, el narratólogo norteamericano, en sus propios intentos de construir un modelo más satisfactorio de gramática de los relatos, propone un desplazamiento hacia paradigmas generativo-transformacionales basándose en la relación de la narrativa con los mecanismos cognitivos de la mente humana:

[T]hough everybody may not know how to narrate effectively, everybody [...] knows how to narrate $[\ldots]$. Furthermore, everybody distinguishes narratives from non-narratives, that is, everybody has certain intuitions [...] about what constitutes a narrative and what does not. [...] In fact, people of widely different cultural backgrounds frequently identify the same given sets as narratives and reject others as non-narratives and they often recount narratives which are very similar. [...] It seems, therefore, that, to a certain extent at least, everybody has the same intuitions - or has internalized the same rules - about the nature of narratives. A grammar of narrative is a series of statements or formulas describing these rules or capable of yielding the same results.

[...] a grammar describes the rules and operations that allow one to process a particular representation as a narrative (if you process $\mathrm{X}$ as a narrative, it is because you make use of the following grammar or of a grammar that is formally equivalent to it). The grammar I will present is [...] strongly inspired by the linguistic grammars described by Noam Chomsky (Prince, 1980: 50).

En la misma vena, aunque con importantes diferencias en sus planteamientos, se encuentran las gramáticas textuales de Dijk (1972), Genot (1976), Pavel (1976) y Ryan (1977), con las que Prince admite estar en deuda (cfr. 1980: 50). En concreto, los trabajos de estos autores representan un nivel de la narratología cognitiva en el que se trata de contestar a la cuestión sobre cómo las estructuras narrativas responden a frames o parámetros cognitivos propios de la mente humana. Estos mismos teóricos y otros que se les han ido sumando han realizado sucesivos ajustes sobre sus esquemas, originados en el estructuralismo y pasados por el filtro de la gramática generativa, en una búsqueda de los modelos lingüísticos que mejor puedan responder a sus propósitos. Entre ellos cabe destacar por su importancia en el desarrollo de la narratología cognitiva los trabajos de Monika Fludernik, Manfred Jahn y Ansgar Nünning, todos ellos profesores del ámbito germano. 
En Towards a "Natural" Narratology (1996), Fludernik se aproxima a la narrativa desde una perspectiva gradualista de acuerdo con la cual una cualidad común subyace de manera natural a todo el espectro continuo que abarca desde las simples anécdotas contadas espontáneamente en comunicaciones interpersonales inmediatas hasta las narraciones más sofisticadas y meditadas. La autora resume qué es la narratología natural como sigue:

\footnotetext{
The term natural feeds from three areas of research [...] Natural linguistics and frame theory enter at several levels of the model $[\ldots]$ my third inspiration $[\ldots]$ has come from Jonathan Culler's concept of naturalization, which I have rechristened narrativization. Narrativization, that is the recognization of a text as narrative, characterizes a process of interpretation by means of which texts come to be perceived as narratives $[\ldots]$ aspects one and two of this schema of natural narratology (the experientiality of natural narrative and the cognitive parameters inherent in natural linguistics) are subsumed in, and incorporated with, the processes of interpretation which constitute the third aspect or level of natural narratology, narrativization (1996: 235).
}

Lo que más aleja a Fludernik de los presupuestos estructuralistas de la narratología clásica es que, para ella, la perspectiva diacrónica juega un papel fundamental en el desarrollo del modelo, contando con el ilustre precedente de Stanzel ([1979] 1984) en la propia tradición germana. Así, la narratología natural está planteada en términos evolutivo-cognitivistas: "These historical aspects are meant to reflect the phylogenetic spread of storytelling parameters from orality into written narrative, and to document the discovery of the deep-structural potentialities of the art of oral storytelling within the written medium" (Fludernik, 1996: 236).

Nünning comparte con Fludernik una visión constructivista de la narratividad que, en su caso, parte de una revisión del concepto de narrador no fiable que enunciara Wayne Booth: "I have called a narrator reliable when he speaks for or acts in accordance with the norms of the work (which is to say the implied author's norms), unreliable when he does not" (1961: 158-59). En una primera fase, el modelo que desarrolla Nünning (1999) sitúa a este tipo de narrador como el resultado de una combinación de marcas en el texto que ayudan al lector a reconocer su falta de fiabilidad y de la construcción activa de un narrador de tales características por parte del lector a través de una estrategia interpretativa. Sin embargo, posteriormente, Nünning introduce la noción de multiperspectivismo, en virtud de la cual reemplaza por completo 
todo el modelo de autor implícito de Booth por una teoría constructivista de atribución de significado textual (cfr. Fludernik, 2005: 50).

Por su parte, Jahn (1997) toma el concepto de frame, desarrollado originalmente por Marvin Minsky dentro del área de la inteligencia computacional, en el sentido que lo había utilizado ya Menakhem Perry como "the cognitive model that is selected and used (and sometimes discarded) in the process of reading a narrative text" (Jahn, 1997: 442). Entre otras nociones y principios, Jahn también toma prestada la idea de "regla de preferencia" introducida por Ray Jackendoff para explicar los procesos de toma de decisiones: "(usually) a nonnecessary but sufficient condition based on inductive clues, graded judgments, and typicality properties that captures a discrete cognitive decision process" (idem: 446). Con todo ello, Jahn se propone recaracterizar dentro del paradigma cognitivo el modelo clásico de las situaciones narrativas de Stanzel ([1979] 1984), llegando como resultado a un"flexible model of the processing of third-person narrative situations (theoretically also of first-person narrative situations), giving a principled account of the hermeneutic interplay between top-down (frame-determined) and bottom-up (data-determined) cognitive strategies" (Jahn, 1997: 464).

Éste y otros artículos de Jahn (1999), así como los comentados de Fludernik y Nünning responden a un planteamiento que concibe la narratología cognitiva como la puesta al día y el enriquecimiento de la teoría narrativa previa gracias a modelos y otros instrumentos teóricos importados de la lingüística cognitiva, el análisis del discurso o la pragmática lingüística. Esta caracterización de los modelos lingüísticos y cognitivos en el marco de la teoria narrativa contrasta por su timidez con la tesis mucho más ambiciosa que representa David Herman:

[B]oth language theory and narrative theory can be viewed as resources for -or modular components of- cognitive science $[. .$.$] reorganizing the study of language and narrative in$ ways that allow for a new interlocking of methodologies, a new synthesis of research methods and aims [...] Both narratology and linguistics will contribute to rethinking narrative as a strategy for creating mental representations of the world (2002: 5).

Ya en su importante artículo "Narratology as a cognitive science" (2000; revisado en 2001), hacía notar cómo la entonces reciente The MIT Encyclopedia of the Cognitive Sciences carecía de entradas específicas sobre narrativa pero se proponía subsanarlo señalando "representative entries that suggest new possibilities for a 
cognitive approach to narrative analysis" y, recíprocamente, "developments in narrative theory that $[\ldots]$ have the potential to reshape central questions in the cognitive sciences" (2000). A través de un barrido exhaustivo del monumental volumen del MIT, Herman descubre una infinidad de posibilidades de cooperación de ambos campos disciplinares en uno y otro sentido hasta el punto de poner de manifiesto que "narratology, like linguistics, can be recharacterized as a subdomain of cognitive-scientific research" (idem).

Este espíritu es el mismo de su gran aportación a la moderna narratología cognitiva, Story Logic (2002), donde retoma la empresa de construir una gramática de los relatos, si bien inmersa por completo en el seno de las ciencias cognitivas. De este modo, para Herman, el lenguaje funciona como un interfaz crucial entre el relato y la cognición pero el objeto del análisis narrativo es el proceso por el cual los receptores reconstruyen el storyworld (mundo de la historia) transmitido narrativamente. Define los storyworlds como "mental models of who did what to and with whom, when, where, why, and in what fashion in the world to which recipients relocate $[\ldots]$ as they work to comprehend a narrative" (2002: 10). Se trata de una nueva concepción "ecológica" del plano clásico de la histoire que equipara en importancia espacialización y temporalización, a la vez que sustituye la simple causalidad lineal de la cadena de acontecimientos por una red mucho más compleja de relaciones que influye en la representación mental que del mundo de la historia se hace el receptor: "Narrative understanding requires determining how the actions and events recounted relate to what might have happened in the past, what could be happening (alternatively) in the present, and what may yet happen as a result of what already has come about" (idem: 14). El modelo de Herman de comprensión narrativa consta de dos grandes sistemas interrelacionados: los microdiseños narrativos, que funcionan a un nivel relativamente local según lo que el receptor necesita modelar para porciones no demasiado extensas del mundo de la historia (estados, acontecimientos, acciones, roles participantes...); y macrodiseños narrativos, que organizan el mundo de la historia a gran escala (temporalidades, especialización, perspectivas, anclaje textual). Se trata en definitiva, no tanto de una adaptación de los conceptos cognitivos a la narrativa, como de una reformulación de los objetivos de la narratología en el marco general de las ciencias cognitivas. La labor posterior de Herman en solitario o en volúmenes colectivos -como coordinador o colaborador- sigue apuntando hacia la consecución de ese estatus 
científico-cognitivo para la narratología y cabe preguntarse, una década después de su primera publicación, si, tal vez, una próxima edición revisada de The MIT Encyclopedia of the Cognitive Sciences incluirá entradas específicas sobre narrativa.

\section{El estructuralismo como actitud cientifista}

Uno de los puntos de contacto más interesantes entre los enfoques estructuralista y cognitivista en la narratología es que ambos parten de una actitud cientifista a la hora de estudiar los fenómenos narrativos.

Dados los vínculos del cognitivismo con la psicología empírica o las ciencias de la computación, el elemento científico de la narratología cognitiva podría darse por sentado. En el caso del estructuralismo, sin embargo, es necesario hacer un mayor esfuerzo de abstracción para percibir el cientifismo subyacente. Esta dificultad puede radicar en que las áreas académicas que han supuesto los campos de aplicación más conocidos del estructuralismo son ámbitos de las Humanidades y las Ciencias Sociales, como la Lingüística, la Antropología o la Semiótica. Pero los objetos materiales y formales de estudio no deben confundirse con la perspectiva de investigación; en este sentido, podría afirmarse que el estructuralismo "pertenece a la ciencia”, como dice Paul Ricoeur en Hermenéutica y estructuralismo (1975: 36). O, quizá más específicamente, podría afirmarse que el estructuralismo tiene una actitud cientifica ante el estudio de un corpus determinado de hechos culturales, ya sean textos, lenguas o relaciones de parentesco. Jan Christoph Meister se ha referido a $S / Z$, de Roland Barthes, como ejemplo de condena de "the rigid systems of early structuralism for claiming absolute scientific validity" (2003: 202). La naturaleza cientifista del estructuralismo ha sido evidenciada asimismo por destacados representantes de esta metodología, como LéviStrauss: "El interés de las investigaciones estructurales es, precisamente, el de darnos la esperanza de que las ciencias más avanzadas (...) pueden proporcionarnos modelos y métodos para solucionar nuestros problemas" (1969: 250).

Una de las características que convierten al estructuralismo en una empresa cientifista es su universalismo. La ciencia (o, al menos, cierta idea de la ciencia) se caracteriza por la búsqueda de leyes universales, más allá de la experiencia y el contexto. Como observaba Karl Popper, el objeto de la ciencia "consiste en dar con 
explicaciones satisfactorias de todo aquello que nos parece precisar una explicación" (1992: 180); explicaciones que deben tender a lo universal para ser satisfactorias. En el Curso de lingüística general, texto fundacional del estructuralismo, Saussure prescribía como una de las tareas de la lingüística "buscar las fuerzas que entran en juego de manera permanente y universal en todas las lenguas" (2006: 31). Es mérito de Saussure haber señalado (aún sin ser el primero en hacerlo) que "el lenguaje no se reduce a la suma de las acciones individuales, sino que constituye una estructura objetiva supraindividual que sigue leyes suprapsicológicas y que es la que posibilita precisamente el desarrollo y ejercicio de los actos lingüísticos individuales" (Velarde Lombraña, 1979: 10. El subrayado es nuestro). "Whatever our ignorance of the language and the culture of the people where it originated, a myth is still felt as a myth by any reader anywhere in the world. Its substance does not lie in its style, its original music, or its syntax, but in the story which it tells", señalaba Lévi-Strauss en "The Structural Study of Myth" (1986: 811); lo cual es especialmente pertinente para nuestro objeto de estudio, al apuntar hacia estructuras narrativas universales (míticas, en este caso), independientes del contexto. Lévi-Strauss, por otro lado, también se ha referido a "la universalidad de la naturaleza humana" en relación a la Antropología (citado en Velarde Lombraña, 1979: 13).

Estrechamente relacionada con el universalismo, la formalización es otra característica científica compartida. Si bien es una característica aplicada a dominios distintos, tanto en el estructuralismo como en el cognitivismo está presente la idea de formular patrones que no estén sujetos a las circunstancias pragmáticas que rodean al objeto de estudio, ya sea un texto narrativo o un sistema de adquisición de conocimiento. Como señala Gilbert Hottois en su Historia de la filosofía del Renacimiento a la Posmodernidad, el estructuralismo es "antihistórico y formal" (1999: 378). La estructura va más allá del sujeto individual; es, según Hjelmslev, una entidad autónoma de dependencias internas. La formalización estructuralista no sólo está presente en la variante lingüística -que reduce los aspectos sustanciales del lenguaje (sustancia fónica y sustancia semántica) a aspectos formales (cfr. Ricoeur, 1975: 154)-, sino también en la antropología de Lévi-Strauss. Las relaciones de la lingüística de Jakobson con la teoría matemática de la comunicación (cfr. Rodrigo Alsina, 1995: 63$65)$ pueden añadirse a los anteriores indicios sobre tendencias formalistas. 
Todo ello implica que el estructuralismo no puede reducirse a un mero descriptivismo empirista o a una metodología puramente taxonómica (cfr. Velarde Lombraña, 1979: 5) ${ }^{5}$; de hecho, el estructuralismo francés rechaza el énfasis en los datos empíricos (cfr. Igartua y Humanes, 2004: 138). E incluso en autores donde puede encontrarse una voluntad taxonómica, como Propp (cfr. Ryan, 1979: 129-130), el sistema que ideó para la morfología del cuento popular ruso "revealed to the next generation some universals of narrative grammar" (Ryan, 1979: 130).

La idea del estructuralismo radica en encontrar estructuras esquemáticas, el "esqueleto" que unifica un corpus de fenómenos diversos. Precisamente en el ámbito de la narratología estructuralista, el proyecto de uno de sus pioneros, Propp, consiste en "the elaboration of schemes and categories that would relate to the actual tales of the corpus as the 'abstract substratum' of grammar relates to a concrete language" (Ryan, 1979: 129). El objetivo del estructuralismo es la reconstrucción de un objeto mediante la manifestación de las reglas de funcionamiento y asociación del objeto (cfr. Barthes, 1983); así, el cientifismo estructuralista puede comprobarse asimismo en su metodología analítica: descomposición de un corpus cerrado de material en elementos y unidades, ubicación de tales elementos o unidades en relaciones de oposición (preferentemente, oposición binaria), y establecimiento de un álgebra o una combinatoria de esos elementos y parejas de oposición. Como dice Ricoeur, "la vía del análisis, de la descomposición en unidades más pequeñas, es la vía misma de la ciencia" (1975: 72).

\section{Narratología estructural y narratología cognitiva}

Aunque tanto el enfoque estructuralista como el enfoque cognitivista suponen proyectos cientifistas, eso no equivale a que compartan la misma metodología, ni a que estén interesados en el mismo objeto de estudio ${ }^{6}$. Incluso, uno de los argumentos contra la

\footnotetext{
${ }^{5}$ A esta imagen meramente descriptivista del estructuralismo ha podido contribuir la opinión de uno de los padres de las ciencias cognitivas, Noam Chomsky, para quien el estructuralismo de Saussure o la glosemática de Hjelmslev forman parte de una lingüística de tipo empirista (cfr. Velarde Lombraña, 1979: 5). Pero, como señala Julián Velarde Lombraña, la "acusación lanzada por Chomsky contra la glosemática (...) no tiene fundamento sólido y en modo alguno es compatible con el sistematismo, exactitud y generalización exigidos por Hjelmslev a toda ciencia” (1979: 5).

6 Para una reflexión sobre las diferencias metodológicas entre el generativismo chomskyano y el estructuralismo saussureano, cfr. Velarde Lombraña, 1979. También hay diferencias conceptuales entre
} 
integración de las teorías cognitivas en la narratología entiende que el estructuralismo, entre otros modelos de recepción, es incompatible con el cognitivismo (cfr. Eders, 2003: 285).

Afortunadamente, existen otros textos de referencia con una opinión algo más matizada. El artículo seminal de Herman, el ya nombrado "Narratology as a cognitive science" (2000), se refiere a clásicos del estructuralismo como Lévi-Strauss o Barthes. Y The MIT Encyclopedia of the Cognitive Sciences establece puentes directos: en la entrada "Semiotics and Cognition" de esta enciclopedia, Patrizia Violi observa que la perspectiva semiótica estructuralista "developed along the lines of Saussure and Hjelmslev (...) is far less interested in issues related to cognition”; sin embargo, Violi destaca precisamente el estudio de la estructura narrativa como un área que presenta similaridades interesantes con las ciencias cognitivas:

Since the seminal work of Vladimir Propp (...), structural semiotics has focused on the analysis of the structural properties of any kind of narrative text, developing a highly complex and articulated model to account for the different levels of structural organization (...). This line of research could be compared to work done in the cognitive area on story grammar (1999: 744).

Por consiguiente, la narratología estructuralista y la narratología cognitiva no sólo compartirían la misma actitud científica básica, sino que sus objetos de estudios serían comparables. Particularmente, el objeto de las story grammars supone un vínculo entre el formalismo estructural y el lenguaje del cognitivismo generativista. Así, David E. Rumelhart observa que las story grammars son esquemas de formalización de una estructura; formalizaciones que usualmente, aunque no siempre, han supuesto "the use of rewrite rules which conveniently, and generatively, capture the relationships among the various pieces of such stories" (1980: 314). El énfasis que pone el estructuralismo en las relaciones no es incompatible con la idea de generación de narrativas; de hecho, en un enfoque cognitivista-generativista de la narratología sería crucial el establecimiento de reglas de generación y transformación de las estructuras sobre las que se arman las narraciones. En esta línea puede encuadrarse la descripción que hace

las ciencias cognitivas y la narratología, como las referentes a las nociones de texto y signo lingüístico (cfr. Meister, 2003: 207-208). Para algunos argumentos en contra de la integración de las teorías cognitivas en la narratología, cfr. Eder, 2003: 285-287. 
Marie-Laure Ryan de la influencia de la gramática generativa chomskyana en la narratología (que, debe recordarse, se desarrolló en un contexto estructuralista):

From Chomsky's linguistic theory, narratology took over this notion of rules endowed with generative power. Drawing a parallel between the native speaker's ability to pass judgments of grammaticality over sentences he has never heard before, and the reader's or listener's ability to tell whether or not a given message can qualify as a story, specialists such as van Dijk (...), Prince (...), and Culler (...) redefined their endeavour as the formulation of the limited set of rules that enables us to produce and recognize an infinite number of stories, and that, consequently, constitutes our narrative competence (Ryan, 1979: 131-132).

También sería comparable la utilización de conceptos instrumentales, como el binding (la representación de conjunciones de propiedades, que está en la base de la capacidad de la representación simbólica), que ha sido relacionado con los actantes de Greimas (Herman, 2000). Por otro lado, existen relaciones históricas directas de influencia entre la narratología de origen formalista y las ciencias cognitivas:

Let us consider the formal description of textual features, which has always been one of the central concerns of narratology. Since Propp, the investigation of formal description has been accompanied by an interest in theories which could employ it in order to model the process of narratio formation. Narratology may therefore have been the first school of literary and textual criticism (as distinct from pure linguistics) to adopt methods and aims which mark it out from the hermeneutic paradigm and give it the theoretical opportunity to draw on the analytic paradigm of empiricism. Moreover, several concepts which originated in narratology (most obviously plot, but also episode) have been adopted by the cognitive sciences; this suggests an affinity between the two disciplines (...) (Meister, 2003: 208).

Desde nuestra perspectiva, el vínculo teórico más interesante entre los objetos de estudio de la narratología estructural y la narratología cognitiva es la idea de que las estructuras y gramáticas narrativas textuales tienen algún tipo de correspondencia isomórfica con los sistemas mentales de procesamiento informativo en formato narrativo; en otras palabras, que existe alguna correspondencia entre las estructuras textuales narrativas y las estructuras cognitivas de recepción de narrativas, lo que permitiría dar un "salto" natural desde el estudio estructural de narraciones hacia las ciencias de la mente. Como ha señalado Louis Tyson en el capítulo sobre "Structuralist criticism" de su Critical Theory Today: A User-Friendly Guide, 
the world we live in consists of innumerable events and objects, that is, innumerable surface phenomena. However, the structures that underlie and organize these phenomena are relatively few. Without these structures our world would be chaos.

Where do these structures come from? Structuralists believe they are generated by the human mind, which is thought of as a structuring mechanism. This is an important and radical idea because it means that the order we see in the world is the order we impose on it. Our understanding of the world does not result from our perception of structures that exist in the world. The structures we think we perceive in the world are actually innate (inborn) structures of human consciousness, which we project onto the world in order to be able to deal with the world. It's not that there is no factual reality; it's that there are too many facts to be perceived without conceptual systems to limit and organize them. And those conceptual systems originate within human consciousness (Tyson, 2006: 210-211).

Hasta cierto punto, la vinculación del texto narrativo y su recepción podría considerarse una necesidad operativa para el estructuralismo. Como señala Jens Eder: "Reception theories are a necessary part of a framework of pragmatic semiotics. Without them, the connection between signs and represented objects is obscured and important structures are hidden from view" (2003: 286). En este sentido, el cognitivismo amplía el abanico instrumental de la narratología: "By integrating cognitive theories into narratology, narratologists are no longer compelled to define narrative phenomena exclusively using purely structural features. Instead, they can combine structural features with functional, reception-dependent features, and that has advantages for the applications of narratological categories" (Eder, 2003: 292); concretamente, esta expansión analítica de la narratología puede revelar estructuras de la recepción: "Cognitive theories give narratology access to new kinds of narrative elements and structures (e.g. structures of character and emotional influence) and potential new ways of defining and explaining narrative phenomena" (Eder, 2003: 295296).

Aunque la idea no es nueva, la narratología no ha abrazado hasta fechas recientes el estudio cognitivo del receptor. Así, Meister se refiere a

an important idea suggested by the grandfather of literary criticism, Aristotle himself, the idea that there is a systematic connection between poetics and rhetoric, in other words, between the aesthetics of production and the aesthetics of effect. Such a synthetic combined 
perspective was in fact practically ignored in most narrative theories before Genette. The theory and aesthetics of reception and the historical trends in the reception of a text are not conventionally seen as narratological topics, and, until only a few years ago, it was taken for granted that the cognitive processing of narratives is the concern of cognitive theory rather than narratology. The situation has of course changed significantly with the recent shift towards constructivism in narrative theory, which is perhaps best demonstrated by Herman (...), who suggests that we should reconceptualize narratology as a cognitive science (2003: 204).

Es interesante señalar que el estructuralismo ya contenía de algún modo la posibilidad de establecer puentes con el estudio de la mente. No es casual, por ejemplo, que Lévi-Strauss haya apuntado lazos con la psicología de la Gestalt (cfr. Lévi-Strauss, 1969: 290-291), o que pensase que "we may be able to show that the same logical processes operate in myth as in science, and that man has always been thinking equally well” (1982: 822). En su diccionario Semiótica, A. J. Greimas y J. Courtés consideran la "competencia semio-narrativa" como "formas —clasificadoras y programadoras- de inteligencia humana"; unas formas que serían, además, universales (1982: 128), y que conforman "el repertorio de las formas susceptibles de ser enunciadas" (1982: 130). Y Umberto Eco usa el término estructura en el Tratado de semiótica general (2000: 69, nota 5) como un modelo que, si funciona, reproduce de algún modo "un orden objetivo de los hechos o un funcionamiento universal de la mente humana".

El "salto" desde una narratología estructural a una narratología cognitiva puede verse en investigaciones como "Cognitive Structures in Comprehension and Memory of Narrative Discourse", donde Perry W. Thorndyke propone un modelo "that attempts to distinguish the structure of a prose passage from its content. The term "structure" is used here to mean the syntax of plot organization in a narrative; that is, the grammar for describing the legal combinations of abstract narrative elements" (1977: 77-78). El objetivo habitual de una "gramática" estructural abstracta de textos narrativos se conjuga con objetos de investigación psicológicos, dado que Thorndike pretende demostrar que las estructuras comunes a un tipo de discursos narrativos "are used during the comprehension and recall of narratives as a technique for improving memory for the text" (1977: 78-79) ${ }^{7}$. Estas facilidades cognitivas se asientan en conceptos como

\footnotetext{
${ }^{7}$ El citado artículo de Thorndyke incluye un estudio experimental que evidencia, efectivamente, "the importance of an identifiable organizational structure for comprehension and memory of narrative discourse" (1977: 95).
} 
el de frame, compuesto por elementos abstractos de una historia que se actualizan en la mente ante la recepción de los materiales empíricos específicos de una historia: "During comprehension of a story, the frame for general "stories" produces a description of the current story by substituting real properties of the story for prototypical ones provided by the frame. This instantiation of a frame is similar to creation of a token node from a type node in a semantic network" (Thorndike, 1977: 83). Enfoques como el de Thorndyke, en resumen, redundan en la idea de que existe algún principio isomórfico entre las estructuras narrativas textuales y las estructuras narrativas mentales; como dice el propio autor en la discusión de su paper, "it is clear from the data presented here that any adequate accounting of discourse comprehension consider the high-level structural characteristics of the text and the reader's internal representation of that information" (Thorndyke, 1977: 105).

La relación entre estructuras narrativas y cognición también puede inferirse a partir del modelo narratológico, basado en la semántica generativa, de Marie-Laure Ryan (cfr. 1979), para quien el modelo supone "a screen through which information is filtered to either produce or interpret narratives and stories" (1979: 141). Es llamativo que David Herman se refiera a esta investigación como "Ryan already suggesting ways to refine strategies for refining what was itself a rethinking of structuralist narratology!" $(2000)^{8}$.

\section{El horizonte neurofisiológico de la narratología}

La narratología cognitiva implica la apertura de una vía de fundamentación científica empírica del estudio de las estructuras narrativas. Eder observa que

cognitivism is in no way fundamentally incompatible with other approaches in the first place: unambiguous efforts to integrate cognitive theories can be found in psychoanalysis and structuralism. This cannot be mere coincidence-cognitivism may well be suited to explaining concepts of semiotics, phenomenology, and the aesthetics of reception and making them compatible with empirical methods (2003: 286-287).

\footnotetext{
${ }^{8}$ El texto de Ryan contiene una crítica al intento de Gerald Prince de apoyarse en la gramática generativotransformacional de Noam Chomsky (Ryan, 1979; Herman, 2000).
} 
Científicamente, el cognitivismo supone un paso adelante respecto al estructuralismo, el cual, aun persiguiendo patrones universales, estaba sujeto (en su vertiente lingüística al menos) al problema de la arbitrariedad del signo (cfr. Saussure, 2006: 182-164). Las ciencias cognitivas aportan una característica que implica un grado de cientificidad superior al del estructuralismo: la naturalización de los procesos mentales. El cognitivismo se aleja de las determinaciones culturales y pretende fundar neurofisiológicamente el lenguaje y los procesos mentales, dado que "es necesario invocar la maduración biológica del substrato neurológico para explicar el desarrollo progresivo de las facultades cognitivas" (Mehler y Dupoux, 2003: 118). Esta fundamentación neurofisiológica de lo cognitivo resulta especialmente relevante si se considera que se ha apuntado la necesidad de matizar la tendencia excesivamente funcionalista que presenta la perspectiva computacional clásica del cognitivismo. Para esta tendencia funcionalista, la información captada por el organismo es transformada en representaciones que permiten "computar una conducta adaptada a la realidad. Todas las operaciones relevantes se realizan sobre esas representaciones, de manera que un sistema cognitivo puede entenderse prescindiendo del sistema nervioso y de las vías que captan la información de la realidad física" (Colom Marañón, 1993: 92). Frente a esto, habría que tener en cuenta las restricciones orgánicas impuestas por el cerebro.

Dado que las ciencias cognitivas apuntan hacia la localización neurofisiológica de los procesos mentales, y dado al mismo tiempo que la narrativa parece ser una función universal de la mente humana, presente en todas las culturas (cfr. Prince, 1980: 50), la idea de restricciones impuestas por el cerebro puede ser iluminadora para un enfoque cognitivista de la narratología, que tendría que tener en cuenta el soporte físico (el cerebro) donde se realizan las computaciones "narratológicas". Esta aproximación neurofisiológica a la narratología debe contextualizarse en el ámbito más general de los estudios de la imagen, donde también se ha propuesto un enfoque neurológico que va más allá de la dicotomía entre mímesis y convencionalismo (Neiva, 2007). Particularmente, resulta destacable que la investigación neurobiológica haya encontrado indicios de correlatos fisiológicos de la función narrativa en el cerebro humano. Uno de esos indicios es que el córtex prefrontal (un área del cerebro envuelta funcionalmente en la cognición) está interconectado con otra región cerebral, el área gnóstica, que sintetiza inputs relativos a situaciones y envía evaluaciones holísticas de las mismas al córtex prefrontal; lo interesante es que, en lo relativo a la memorización de la experiencia, tras 
la interacción con el córtex el área gnóstica almacena la experiencia en formato narrativo ("story form") (Beatty y McCroskey, 2001: 103-104). Se produce, así, una vinculación entre cognición y narratividad en áreas funcionales del cerebro que puede abrir prometedoras vías de investigación empírica.

\section{Conclusiones}

La narratología cognitiva supone la posibilidad de superar la separación entre texto (objeto de estudio externo) y mente (objeto de estudio interno), dado que una fusión colaborativa entre la tradición formalista-estructuralista y el giro cognitivista en narratología podría revelar que las estructuras narrativas inferibles de un corpus textual determinado pueden mantener relaciones isomórficas con sistemas cognitivos de procesamiento de información en formato narrativo; quizá, hasta el punto de trabajar en una dirección guiada por "the theoretical stance that stories constitute the unit of data processed by human neurobiological coding of experience" (Beatty y McCroskey, 2001: 118). Esta postura teórica puede ser muy extrema, pero apunta a una vía de investigación cuanto menos prometedora, dados los indicios de solidez que presenta la narratología cognitiva.

\section{Bibliografía}

BARTHES, Roland (1966); "Introduction a l'analyse structurale des récits", en Communications, 8 (1966), pp. 1-27.

BARTHES, Roland (1983): "La actividad estructuralista", en Ensayos críticos. Traducción castellana de Carlos Pujol. Seix Barral, Barcelona, pp. 255-262.

BEATTY, Michael J., y McCROSKEY, James C. (2001): The Biology of Communication. Hampton Press, Creskill (New Jersey). 
BOOTH, Wayne (1961): The Rhetoric of Fiction. University of Chicago, Chicago.

BREMOND, Claude (1966): "La logique des posibles narratifs", en Communications, 8 (1966), pp. 60-76.

BREMOND, Claude (1973): Logique du récit. Seuil, París.

COLOM MARAÑÓN, Roberto (1993): “¿Sabe geometría el martín pescador?: Hacia una concepción psicológica del conocimiento humano", en Estudios de Psicología, 1993, 50, pp. 89-106.

CULLER, Jonathan (1975): Structuralist Poetics: Structuralism, Linguistics, and the Study of Literature. Cornell University Press, Nueva York.

DIJK, Teun A. van (1972): Some Aspects of Text Grammars: A Study in Theoretical Poetics and Linguistics. Mouton, La Haya.

DOLEZEL, Lubomir (1990): Occidental Poetics: Tradition and Progress. University of Nebraska Press, Lincoln.

ECO, Umberto (1966): “James Bond: une combinatoire narrative”, en Communications, 8 (1966), pp. 77-93.

ECO, Umberto (2000): Tratado de semiótica general. Traducción de Carlos Manzano. Lumen, Barcelona.

EDER, Jens (2003): "Narratology and Cognitive Reception Theories", en KINDT, Tom, y MÜLLER, Hans-Harald (Eds.): Narratologia: What Is Narratology? Walter de Gruyter, Berlin y New York, pp. 277-301.

FLUDERNIK, Monika (1996): Towards a "Natural” Narratology. Routledge, Londres. 
FLUDERNIK, Monika (2005): "Histories of Narrative Theory (II): A Genealogy of Early Developments", en PHELAN, J. y RABINOWITZ, P. J. (eds.): A Companion to Narrative Theory. Blackwell, Oxford, pp. 36-59.

FLUDERNIK, Monika (2009): An Introduction to Narratology. Routledge, Londres y Nueva York.

GENETTE, Gérard (1966): "Frontières du récit", Communications, 8 (1966), pp. 152163.

GENETTE, Gérard (1972): Figures III. Seuil, París.

GENOT, Gérard (1976): Problemes de calcul du récit. Université Paris X-Nanterre, París.

GREIMAS, A. J. (1966a): "Eléments pour une theorie de l'interpretation du récit mythique", en Communications, 8 (1966), pp. 28-59.

GREIMAS, A. J. (1966b): Semantique structurale. Larousse, París.

GREIMAS, A. J., y COURTÉS, J. (1982): Semiótica. Versión española de Enrique Ballón Aguirre y Hermis Campodónico Carrión. Gredos, Madrid.

HERMAN, David (2000): "Narratology as a cognitive science", en Image [\&] Narrative, Issue 1, September 2000-18. Disponible en Internet (14/09/2009): http://www.imageandnarrative.be/narratology/davidherman.htm

HERMAN, David (2001): "Narrative Theory and the Cognitive Sciences", en Narrative Inquiry, 11:1, pp. 1-34.

HERMAN, David (2002): Story Logic: Problems and Possibilities of Narrative. University of Nebraska Press, Lincoln. 
HERMAN, David (2005): "Histories of Narrative Theory (I): A Genealogy of Early Developments", en PHELAN, J. y RABINOWITZ, P. J. (eds.): A Companion to Narrative Theory. Blackwell, Oxford, pp. 19-35.

HERMAN, David (2009): Basic Elements of Narrative. Wiley-Blackwell, Oxford.

HOTTOIS, Gilbert (1999): Historia de la filosofía del Renacimiento a la Posmodernidad. Traducción de Marco Aurelio Galmarini. Cátedra, Madrid.

IGARTUA, Juan José, y HUMANES, María Luisa [2004]: Teoría e investigación en comunicación social. Síntesis, Madrid.

JAHN, Manfred (1997): "Frames, Preferences, and the Reading of Third-Person Narratives: Towards a Cognitive Narratology”, en Poetics Today, 18: 4 (invierno de 1997), pp. 441-468.

JAHN, Manfred (1999): "More Aspects of Focalization: Refinements and Applications", en GRAAT (Groupes de Recherches Anglo-Américaines de Tours), 21 [“Recent Trends in Narratological Research"], pp. 85-110.

KINDT, Tom y MULLER, Hans-Harald (2003): "Preface", en KINDT, Tom y MULLER, Hans-Harald (eds): Narratologia: What Is Narratology? Questions and Answers Regarding the Status of a Theory. Walter de Gruyter, Berlín \& Nueva York, pp. v-vii.

LÉVI-STRAUSS, Claude (1958): Anthropologie structurale. Plon, París.

LÉVI-STRAUSS, Claude (1969): Antropología estructural. Traducida por: Eliseo Verón. Revisor técnico: Eduardo Luis Menéndez. Segunda edición. Eudeba (Editorial Universitaria de Buenos Aires).

\footnotetext{
${ }^{9}$ Fecha del Depósito legal.
} 
LÉVI-STRAUSS, Claude (1986): "The Structural Study of Myth", en ADAMS, Hazard, y SEARLE, Leroy (editors): Critical Theory Since 1965. University Press of Florida, Tallahassee, pp. 808-822.

LUNTLEY, Michael (2003): Wittgenstein: Meaning and Judgment. Blackwell, Oxford.

MEHLER, Jacques, y DUPOUX, Emmanuel (2003): Nacer sabiendo. Versión española de Nuria Sebastián. Alianza, Madrid.

MEISTER, Jan Christoph (2003): Narratologia: Computing Action. Translated by Alastair Matthews. Walter de Gruyter, Berlín y Nueva York.

METZ, Christian (1966): “La grande syntagmatique du film narratif”, en Communications, 8 (1966), pp. 120-124.

MILLNER, Andrew y BROWITT, Jeff (2002): Contemporary Cultural Theory: An Introduction. Allen \& Unwin, Crownest (Australia).

NEIVA, Eduardo (2007): "The serpent's egg: Communication and the bio-semiotics of image making”, en Semiotica 167 - 1/4 (2007), pp. 193-222.

NÜNNING, Ansgar (1999): "Unreliable, Compared to What?: Towards a Cognitive Theory of Unreliable Narration: Prolegomena and Hypotheses", en Grünzweig, W. y Solbach, A. (eds.): Grenzüberschreitungen: Narratologie im Kontext/Transcending Boundaries: Narratology in Context. Gunter Narr, Tübingen, pp. 53-74.

PAVEL, Thomas (1976): La Syntaxe Narrative des Tragedies de Corneille. Klincksieck, París.

PHELAN, James y RABINOWITZ, Peter J. (eds.) (2005): A Companion to Narrative Theory. Blackwell, Oxford. 
POPPER, Karl (1992): "El objeto de la ciencia”, en Conocimiento objetivo. Traducción de Carlos Solís Santos. Tecnos, Madrid, pp. 180-192.

PRINCE, Gerald (1973): A Grammar of Stories. Mouton, La Haya.

PRINCE, Gerald (1980): “Aspects of a Grammar of Narrative”, en Poetics Today, 1:3 ("Narratology I: Poetics of Fiction", primavera de 1980), pp. 49-63.

PRINCE, Gerald (2003): "Surveying Narratology", en KINDT, Tom y MULLER, Hans-Harald (eds): Narratologia: What Is Narratology? Questions and Answers Regarding the Status of a Theory. Walter de Gruyter, Berlín y Nueva York, pp. 1-16.

PROPP, Vladimir (1998): Morfología del cuento. Akal Ediciones, Madrid.

RICOEUR, Paul (1975): Hermenéutica y estructuralismo. Traducción al castellano: Graziella Baravalle y María Teresa La Valle. Ediciones Megápolis / Asociación Editorial La Aurora, Buenos Aires.

RODRIGO ALSINA, Miquel (1995): Los modelos de la comunicación. Segunda edición. Tecnos, Madrid.

RUMELHART, David E. (1980): “On Evaluating Story Grammars”, en Cognitive Science, 4, 1980, pp. 313-316.

RYAN, Marie-Laure (1977): "Narration, génération, transformation: La Grande Bretèche de Balzac", en L'Esprit Créateur, 17, pp. 195-210.

RYAN, Marie-Laure (1979): “Linguistic Models in Narratology: From Structuralism to Generative Semantics", en Semiotica Volume 28-1/2, 1979, pp. 127-155.

SAUSSURE, Ferdinand de (2006): Curso de lingüistica general. Traducción castellana y notas de Mauro Armiño. Akal, Tres Cantos (Madrid). 
SHKLOVSKY, Viktor (1990): Theory of Prose. Dalkey Archive Press, Elmwood Park (Illinois).

STANZEL, F. K. (1984): A Theory of Narrative. Cambridge University Press, Cambridge.

THORNDYKE, Perry W. (1977): "Cognitive Structures in Comprehension and Memory of Narrative Discourse”, en Cognitive Psychology, 9 (1977), pp. 77-110.

TODOROV, Tzvetan (1966): “Les catégories du récit littéraire”, en Communications, 8 (1966), pp. 125-151.

TODOROV, Tzvetan (1969): Grammaire du Décameron. Mouton, La Haya.

TOMAN, J. (1995): The Magic of a Common Language: Jakobson, Mathesius, Trubetzkoy, and the Prague Linguistic Circle. The MIT Press, Cambridge (Massachussets).

TOMASHEVSKY, Boris (1965): “Thematics”, en LEMON, L. T. y REIS, M. J. (eds.): Russian Formalist Criticism. University of Nebraska Press, Lincoln, pp. 61-95.

TYSON, Louis (2006): Critical Theory Today: A User-Friendly Guide. Routledge, Londres y Nueva York.

VELARDE LOMBRAÑA, Julián (1979): “Metodología de la gramática generativa”, en El basilisco, número 7, mayo-junio 1979, pp. 4-15.

VIOLI, Patrizia (1999): “Semiotics and Cognition”, en WILSON, Robert A., y KEIL, Frank C. (Eds.): The MIT Encyclopedia of the Cognitive Sciences. The MIT Press, Cambridge (Massachussets) y Londres, pp. 744-745.

WILSON, Robert A. y KEIL, Frank C. (eds.) (1999): The MIT Encyclopedia of the Cognitive Sciences. The MIT Press, Cambridge (Massachussets) y Londres. 
